\title{
IMPAIRED LYMPHOCYTE FUNCTION IN DEPRESSIVE ILLNESS
}

\author{
Ziad Kronfol* Joseph Silva, Jr., John Greden, Susan Dembinski, \\ Robert Gardner and Bernard Carroll
}

Departments of Psychiatry and Internal Medicine University of Michigan Medical Center, Ann Arbor, Michigan

(Received in final form May 2, 1983)

\section{Summary}

Mood states and immunity may be related. To investigate the immune status of patients with primary depressive illness, we compared in-vitro lymphocytic responses to three different mitogens in 26 drug-free depressed patients and 20 normal controls of comparable age and sex. We observed a generalized and marked decrease in the lymphocyte mitogenic activity among the depressive group. This defect in lymphocyte function may be indicative of an impairment in cell-mediated immunity in patients with primary depressive illness.

For more than 2000 years, clinicians have observed that mood states may affect susceptibility to physical illness. In 200 A.D., the Greek scientist Galen noted that melancholic women developed breast cancer more frequently than sanguine women (1). Recent epidemiologic studies tend to support this conclusion (2). Depression may be associated with a variety of medical illnesses including cancer (3), infection (4) and allergic disorders (5), conditions reportedly linked to derangements in immune function. The immune status of patients with affective disorders, however, has not been systematically investigated. Preliminary studies suggest that psychosocial stressors such as bereavement (6) and sleep deprivation (7) may be accompanied by an impairment in cell-mediated immunity. To address this issue in affective disorders, we studied in-vitro lymphocytic response to mitogen stimulation in patients with primary depressive illness and normal controls. We observed a generalized and marked reduction in the mitogenic activity among the depressive group.

\section{Subjects}

Two groups of subjects were studied: depressed patients $(n=26)$ and normal controls $(n=20)$. All patients have been evaluated at the Clinical Studies Unit, a research unit of the Department of Psychiatry at the University of Michigan Medical Center with special interest in affective disorders. The mean age of the depressed group was $41.69 \pm 14.44$ (range:20-68) years. Eight patients were male, 18 female. All patients met the Research Diagnostic Criteria (RDC) (8) for major depressive disorder, primary subtype. Patients had a mean score of $16.26 \pm 6.47$ on the 17-item Hamilton Depression Rating Scale (HDRS) (9). The normal control group was composed of healthy euthymic volunteers. Their mean age was $36.60 \pm 14.90$ (range:21-66) years. Eight

\footnotetext{
*Current Address: Department of Psychiatry

University of Iowa College of Medicine

500 Newton Road, Iowa City, Iowa 52242
} 
subjects were male, 12 female. All patients and controls were free of medical illnesses known to interfere with the reactivity of lymphocytes in culture and were drug-free for at least two weeks prior to the immune testing. Informed consent was obtained from all participating subjects.

\section{Methods}

Twenty milliliters of venous blood were collected from each subject at 8:00 a.m., anticoagulated with 100 units of preservative-free sodium-heparin and centrifuged over Ficoll-Hypaque at $400 \mathrm{x} \mathrm{g}$. for 40 minutes. A lymphocyte-rich fraction was extracted and washed with phosphate buffered saline $(\mathrm{pH}: 7.4)$. Lymphocytes were resuspended in a mixture of RPMI-1640 tissue culture medium containing $20 \%$ autologous plasma and 100 units $/ \mathrm{ml}$ penicillin $\mathrm{G}$ and $100 \mathrm{mg} / \mathrm{ml}$

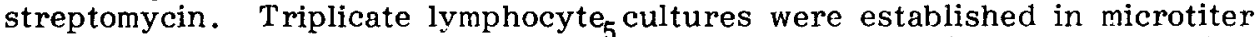
plates. To each well were added $10^{5}$ small lymphocytes (without correction for monocyte contamination) and one of the following mitogens: Phytohemagglutinin$P$ (PHA) (Difco Inc., Detroit, Michigan) in doses of $3.125,6.25,12.5,25$ or $50 \mathrm{\mu g} / \mathrm{ml}$; Concanavalin A (Con A) (Sigma Chemiculs, St. Louis, Missouri), $10 \mu \mathrm{g} / \mathrm{ml}$; or Pokeweed Mitogen (PWM) (Gibco, Grand Island, New York), 1:10 dilution. Lymphocytes from all subjects were also cultured without mitogen to determine background transformations.

All cultures were incubated at $37^{\circ} \mathrm{C}$ in a $5 \frac{\circ}{\circ} \mathrm{CO}_{2}$ humidified atmosphere. After 96 hours of incubation, $1 \mu \mathrm{Ci}$ of tritiated thymidine (specific activity, 20 $\mathrm{Ci} / \mathrm{mmole}$ ) was added to each well and incubation was continued. Six hours later, the cultures were harvested onto Whatman glassfiber filter paper with a multiple-sample precipitator. Filter papers were washed 8-10 times with $0.85 \%$ $\mathrm{NaCl}$ and air-dried. Samples were then immersed in toluene and the radioactivity measured with a liquid scintillation counter.

\section{Calculations and Statistical Analysis}

The arithmetic means of the triplicate samples were calculated and expressed as scintillation counts per minute (cpm). Mitogen response was then recorded as the difference, in cpm, between stimulated and background (unstimulated) counts. The data was then statistically analyzed with one-way analysis of variance (ANOVA) for intergroup comparisons. For PHA, since a dose-response curve was available, we compared the areas under the curves for the two groups. For Con A and PWM, comparisons were made for the specific concentrations used.

\section{Results}

Figure 1 shows the PHA dose-response curves for the depressed and control groups. There was a reduction in lymphocyte mitogenic activity among the depressed group at all concentrations of PHA used. The area under the curve for the depressed group was $1289158 \pm 104337$ units $(\mathrm{cpm} \mathrm{x} \mu \mathrm{g} / \mathrm{ml})$, compared to $1757939 \pm 118963$ units for the control group. This difference was highly significant $[F(1,44)=8.76 ; p=.0049]$. Since the largest difference in lymphocyte responses to PHA occurred at the concentration of $25 \mu \mathrm{g} / \mathrm{ml}$, this concentration was used for further intergroup comparisons of recorded test values. Table I summarizes the results. Lymphocyte responses among the depressed group were markedly reduced with all three mitogens used. The results were statistically significant for PHA $[F(1,44)=11.21 ; p=.0017]$, Con A $[F(1,44)=10.43 ; p=.0023]$ and PWM $[F(1,44)=17.30 ; p=.0001]$. 


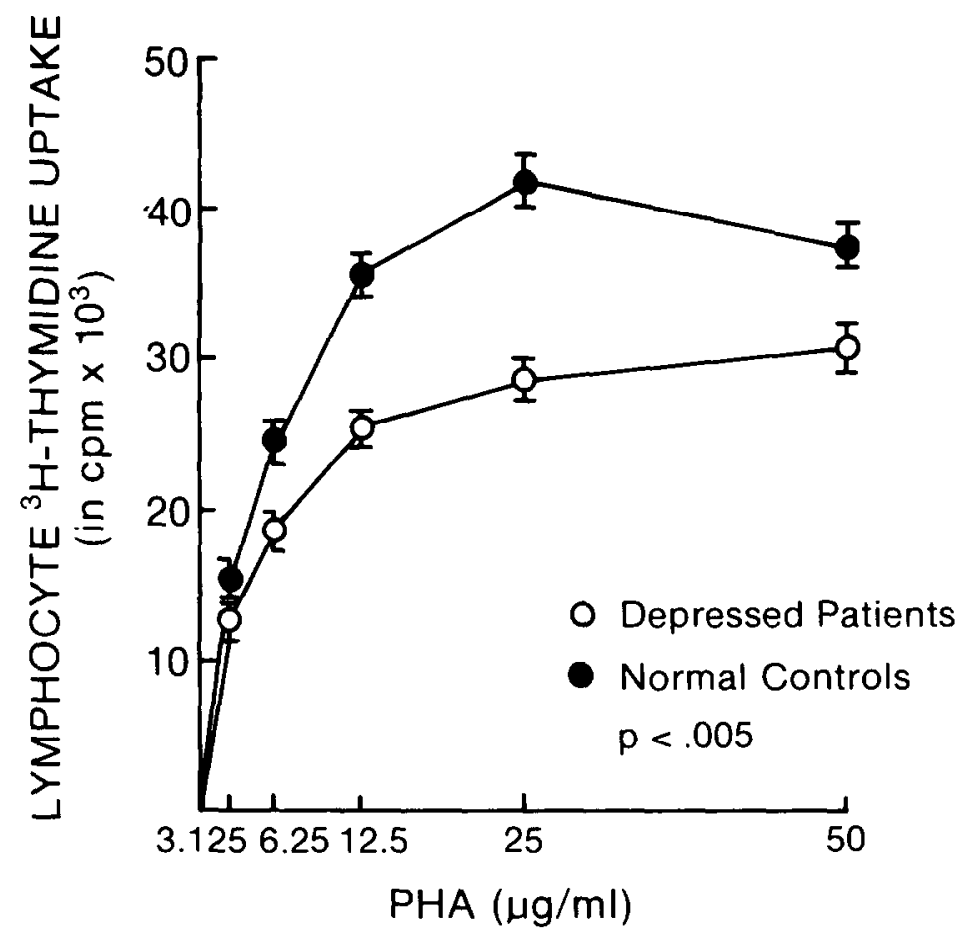

FIG. 1

PHA dose-response curves of peripheral blood lymphocytes from depressed patients and normal controls. Values represent $\mathrm{cpm}$ (mean \pm SEM), indicative of $\mathrm{H}$-Thymidine incorporation into lymphocytes.

PHA = Phytohemagglutinin $-\mathrm{P} ; \mathrm{cpm}=$ scintillation counts per minute ${ }^{*} p<0.005$, areas under the curve, ANOVA.

\section{TABLE I}

In-Vitro Lymphocytic Responses to Mitogen Stimulation in Depressed Patients and Normal Controls

\begin{tabular}{lcccc}
\hline $\begin{array}{l}\text { Mitogen } \\
\text { Tested* }\end{array}$ & $\begin{array}{c}\text { Depressed Patients } \\
(\mathrm{n}=26)\end{array}$ & $\begin{array}{c}\text { Normal Controls } \\
(\mathrm{n}=20)\end{array}$ & $\begin{array}{c}\text { F Values } \\
(1,44)\end{array}$ & $\begin{array}{c}\text { Probability Values } \\
\text { (two-tailed })\end{array}$ \\
$\begin{array}{l}\text { PHA } \\
25 \mu \mathrm{g} / \mathrm{ml}\end{array}$ & $27,904 \pm 2,659$ & $41,410 \pm 3,032$ & 11.21 & .0017 \\
$\begin{array}{l}\text { CON A } \\
10 \mu \mathrm{g} / \mathrm{ml}\end{array}$ & $29,462 \pm 3,768$ & $47,938 \pm 4,296$ & 10.43 & .0023 \\
$\begin{array}{l}\text { PWM } \\
1: 10\end{array}$ & $26,531 \pm 3,463$ & $48,370 \pm 3,948$ & 17.30 & .0001 \\
\hline
\end{tabular}

*PHA, Phytohemagglutinin-P; CON A, Concanavalin A; PWM, Pokeweed Mitogen. The data are expressed as scintillation counts per minute (arithmetic means \pm SEM). 
We then examined the relation between severity of depression and lymphocyte transformations in the depressive group. Because the largest differences in lymphocyte responsiveness between the depressed and control groups were observed with PWM, responses to this mitogen were used to study the effects of depression severity. There was a weak negative correlation $(r=-0.15 ;$ N.S. $)$ between lymphocyte responses to PWM and HDRS scores in the depressive group (Figure 2).

We further divided the depressed patients, on the basis of their HDRS scores, in three subgroups: patients with a mild depression (HDRS < 12), patients with a moderate depression (HDRS=12-20) and patients with severe depression (HDRS $>20$ ). Figure 3 graphically illustrates the results. All three subgroups of depressed patients had significantly lower lymphocyte responses to PWM than the control group ( $p<.05$, ANOVA). There was a trend, among depressed patients, for the lymphocyte stimulation counts to decrease with increasing severity of depression. The differences between the three depressed subgroups, however, were not statistically significant.

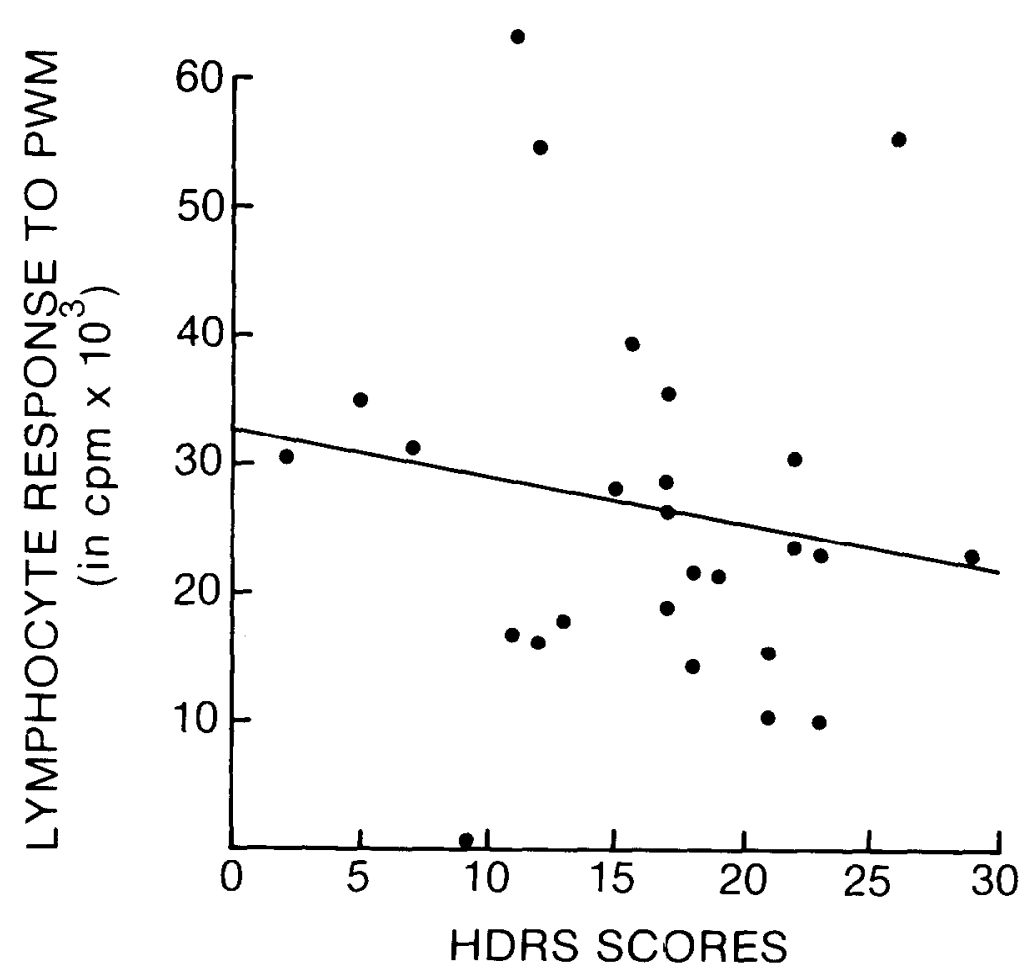

FIG, 2

Scatter Plot showing relationship between lymphocyte responses to PWM and HDRS scores in depressed patients.

PWM $=$ Pokeweed Mitogen

HDRS = Hamilton Depression Rating Scale

$\mathrm{cpm}=$ counts per minute 


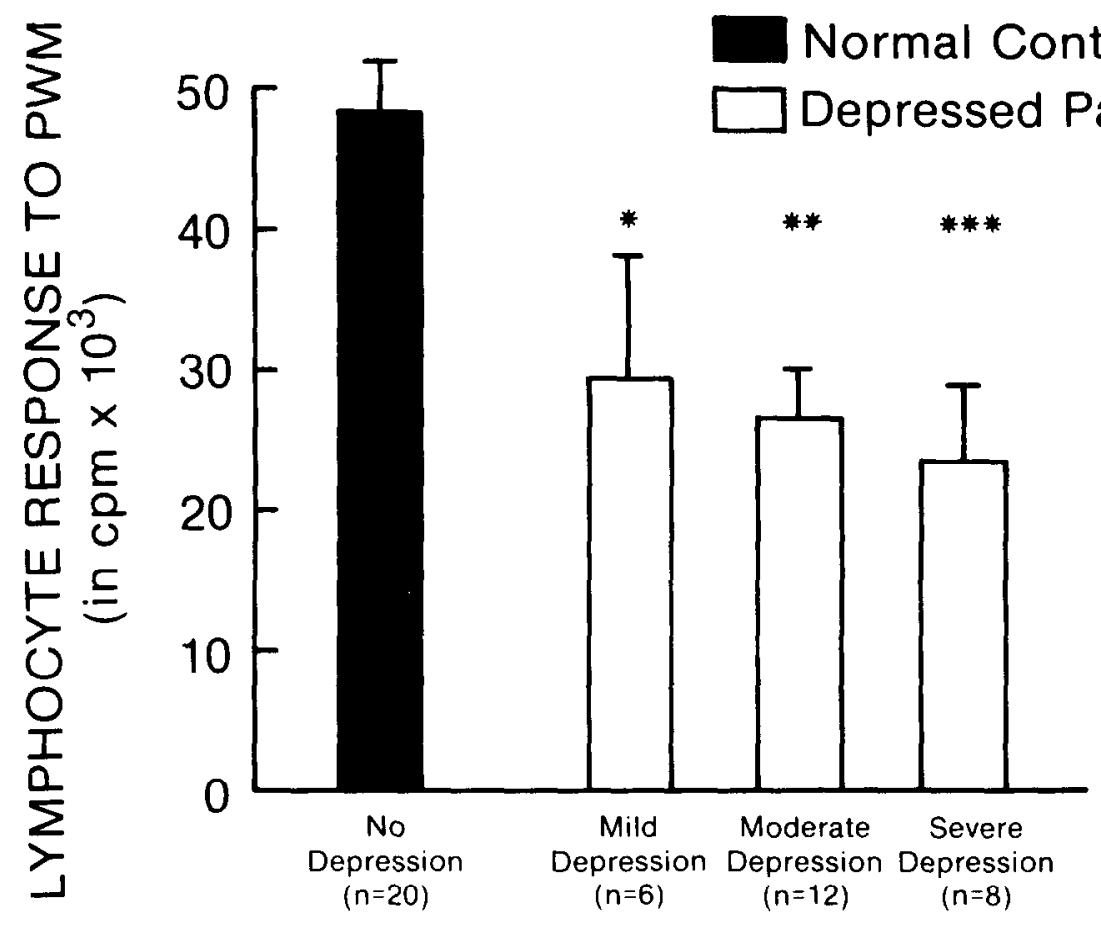

FIG. 3

Lymphocyte Response to PWM in depressed patients and normal controls. The data are expressed in cpm (arithmetic means \pm SEM).

PWM $=$ Pokeweed Mitogen.

Comparisons are made between the normal control group and each of the depressive subgroups.

$\begin{aligned} * p & <0.05 \\ * * p & <0.01 \\ * * * p & =0.001\end{aligned}$

\section{Discussion}

A number of factors known to interfere with in-vitro lymphocytic responses to mitogen stimulation (10) should be considered in interpreting our results. Most relevant are nutritional factors (11), medical illness and drugs (10), diurnal variation (12) and age (13). Our patients were not undernourished (mean weight: $67.46 \mathrm{kgs}$ ) and all subjects with serious medical illnesses were excluded, All subjects were drug-free for at least two weeks prior to immune testing and patients who were receiving medications prior to this drug-free wash-out period did not differ significantly from those who were unmedicated. All blood samples were drawn at the same time of the day to control for possible diurnal variation, and all assays were performed the same day the blood was drawn. Age was slightly higher among the depressed group, but the difference was not statistically significant $(t=1.145 ; p=.25 ; N . S$.$) . The female-to-male$ ratio mildly favored the depressed group, but again the sex differences were 
not statistically significant (Fisher's exact $\mathrm{p}=.1985$; N.S.). Furthermore, in separate ANOVA and ANCOVA models (14) with agc and sex as additional sources of variations between the groups, the basic differences in mitogenic activity between the depressed and control groups remained essentially unchanged $(p \leq .01)$ for all three mitogens used.

Lymphocyte response to mitogen stimulation is a direct measure of in-vitro lymphocyte functional activity and a correlate of cell-mediated immunity. PHA and Con A stimulate mostly T-lymphocytes, the mediators of cellular immunity of the delayed type, while PWM stimulates primarily B-lymphocytes, the cells that mediate the production of immunoglobulins. Both T-lymphocytes and B-lymphocytes are active in protecting the organism against infection. Moreover, $\mathrm{T}$ lymphocytes mediate graft rejection and are also theorized to provide immune surveillance against cancer, whereas B-lymphocytes play a major role in autoimmune diseases.

A decrease in in-vitro lymphocyte functional activity may be indicative of an impairment in cell-mediated immunity. The significant reductions in the mitogenic responses to PHA, Con A and PWM in the depressed group suggest a generalized impairment of immune function in patients with this illness. Whether such an impairment contributes to the increased vulnerability of depressed patients to medical illnesses such as infection (15) and neoplasm (16) remains to be determined. Depressed patients are also known to be hypersecretors of cortisol (17). The effect of hypercortisolemia on the overall immune status of depressed patients is currently under investigation. Furthermore, there have recently been reports of a possible association between HI.A and depression (18). Our findings of impaired immunity in depressive illness thus suggest a complex interface between genetic, immune, neuroendocrine and behavioral regulation, and open the way for future research into the "psychoneuroimmunology" of depressive disorders.

\section{Acknowledgments}

This work was supported in part by PHS Grant MH 28294. We thank the staff of the Clinical Studies Unit for valuable assistance at different stages of the project. Kice Brown, M.S. helped with statistical analysis and Susan G. Heitshusen typed the manuscript.

\section{References}

1. C. B. BAHSON, Psychosomatics 21 975-981 (1980).

2. M. ROGERS, D. DUBEY, P. REI CH, Psychosom. Med. 41 147-164 (1979).

3. R. SHEKELLE, W. RAYNOR, A. OSTFELD, D. GARRON, L. BIELIAUSKAS, S. LIU, C. MALIZA, O. PAUL, Psychosom Med 43 117-125 (1981).

4. G. DAY, Lancet I 1025-1028 (1951).

5. S. NASR, G. ALTMAN, H. MELTZER, J. Affective Disorders $\underline{3}$ 291-296 (1981).

6. R. BARTROP, I. LAZARUS, E. LUCKHURST, L. KILOH, Lancet I 834-836 (1977).

7. J. PALMBLAD, B. PETRINI, J. WASSERMAN, T. AKERSTEDT, Psychosom Med 41 273-278 (1979).

8. R. STITZER, J. ENDICOTT, E. ROBINS, New York State Psychiatric Institute (1977).

9. M. HAMILTON, Brit J Soc Clin Psychol 6 278-296, (1967).

10. J. OPPENHEIM, S. DOUGHERTY, S. CHĀN, J. BAKER, Grune and Stratton, 87-109 (1975). 
11. W. BEISEL, R. EDELMAN, K. NAUSS; R. SUSKIND, J Am Med Assoc 245 53-58 (1981).

12. H. TAVADIA, K. Fleming, P. HUME, H. SIMPSON, Clin Exp Immunol 22 190-193, (1975).

13. J. PORTARO, G. GLICK, L. ZIGHELBOIM, Clin Immunol Immunopath 11 339-345 (1978).

14. J.T. HELWIG, K.A. COUNCIL, eds. SAS User's Guide, 1979 Edition. SAS Institute Inc., Raleigh, North Carolina (1979).

15. J. BALDWIN, Psychol Med 9 611-618(1979).

16. F. WHITLOCK, M. SISKIND, Psychol Med 9 747-752, (1979).

17. B. CARROLL, G. CURTIS, B. DAVIES, J. MENDELS, A. SUGERMAN, Psychol Med 6 43-50 (1976).

18. I. WEITKAMP, H. STANCER, E. PERSAD, C. FLOOD, S. GUTTORMSEN, N Engl J Med 305 1301-1306 (1981). 